\title{
Scaling of nano-Schottky-diodes
}

\author{
G. D. J. Smit, ${ }^{\text {a) }}$ S. Rogge, and T. M. Klapwijk \\ Department of Applied Physics and DIMES, Delft University of Technology, Lorentzweg 1, 2628 CJ Delft, \\ The Netherlands
}

(Received 28 February 2002; accepted 20 September 2002)

\begin{abstract}
A generally applicable model is presented to describe the potential barrier shape in ultrasmall Schottky diodes. It is shown that for diodes smaller than a characteristic length $l_{\mathrm{c}}$ (associated with the semiconductor doping level) the conventional description no longer holds. For such small diodes the Schottky barrier thickness decreases with decreasing diode size. As a consequence, the resistance of the diode is strongly reduced, due to enhanced tunneling. Without the necessity of assuming a reduced (non-bulk) Schottky barrier height, this effect provides an explanation for several experimental observations of enhanced conduction in small Schottky diodes. (C) 2002 American Institute of Physics. [DOI: 10.1063/1.1521251]
\end{abstract}

The effect of downscaling the dimensions of a device on its electrical transport properties is an important topic today. Extremely small diodes have been experimentally realized and characterized in various systems, for example, carbon nanotube heterojunctions, ${ }^{1}$ junctions between $p$-type and $n$-type Si nanowires, ${ }^{2}$ or junctions between the metallic tip of a scanning tunneling microscope and a semiconductor surface. ${ }^{3,4}$ These experiments showed several deviations from conventional diode behavior. Despite some modeling in truly one-dimensional systems, ${ }^{5,6}$ little work has been done on modeling the effects of downscaling a conventional diode, in the regime where quantum confinement does not play a role.

In this letter we present a simple model (based on the Poisson equation) describing the barrier shape in a diode, that is readily applicable to arbitrarily shaped small junctions. It is related to descriptions of inhomogeneities in the Schottky barrier height (SBH) in large diodes, ${ }^{7}$ barrier shapes in small semiconducting grains, ${ }^{8}$ and charge transfer to supported metal particles. ${ }^{9}$ Although we restrict ourselves to metal-semiconductor junctions, the model can easily be adapted, for example, to $p-n$ junctions. The main result is that if the size of the metal-semiconductor interface is smaller than a characteristic length $l_{\mathrm{c}}$, the thickness of the barrier is no longer determined by the doping level or the free carrier concentration, but instead by the size and shape of the diode. The resulting thin barrier in small diodes will give rise to enhanced tunneling, qualitatively explaining measurements of enhanced conduction, ${ }^{3,4,10}$ without the necessity of assuming a reduced SBH. Moreover, experimentally observed scaling behavior and deviating $I V$ curve shapes ${ }^{10}$ can be explained.

The transport properties of a Schottky diode are governed by the potential landscape that has to be traversed by the charge carriers. First, we study an easily scalable and highly symmetrical model system, namely a metallic sphere embedded in semiconductor (see Fig. 1, upper left inset). The radius $a$ of the metallic sphere is a measure for the interface size: for large $a$, we expect to find the well-known results for

${ }^{a}$ Electronic mail: g.d.j.smit@tnw.tudelft.nl a conventional diode, while decreasing $a$ gives the opportunity to study finite size effects.

We only model the barrier shape in the semiconductor; the $\operatorname{SBH} \varphi_{\mathrm{B}}$ is accounted for in boundary conditions and is considered as a given quantity. For simplicity, the depletion approximation $^{11}$ is adopted, which is valid for a wide range of realistic parameters. Moreover, the space charge region is assumed to be homogeneously charged, an assumption that will be discussed later. Solving the Poisson equation in $n$-type silicon with the boundary condition that the charge on the sphere cancels the total charge in the space charge region, we find for $0 \leqslant x \leqslant w$,

$$
\frac{e}{k T} \cdot V(x)=\frac{1}{2 L_{\mathrm{D}}^{2}}\left[(a+w)^{2}-\frac{2(a+w)^{3}}{3(a+x)}-\frac{(a+x)^{2}}{3}\right],
$$

where $x$ is the radial distance from the interface, $w$ is the depletion width, and $L_{\mathrm{D}}=\sqrt{\varepsilon_{\mathrm{s}} k T /\left(e^{2} N_{\mathrm{d}}\right)}$ the Debye length. The zero-point of the potential is chosen in the semiconductor bulk. The value of $w$ is fixed by the second boundary condition $V(0)=V_{\mathrm{s}}$, where $V_{\mathrm{s}}$ is the total potential drop over the space charge region and satisfies $V_{\mathrm{s}}=\left(\varphi_{\mathrm{B}}-\varphi_{\mathrm{s}}\right) / e$

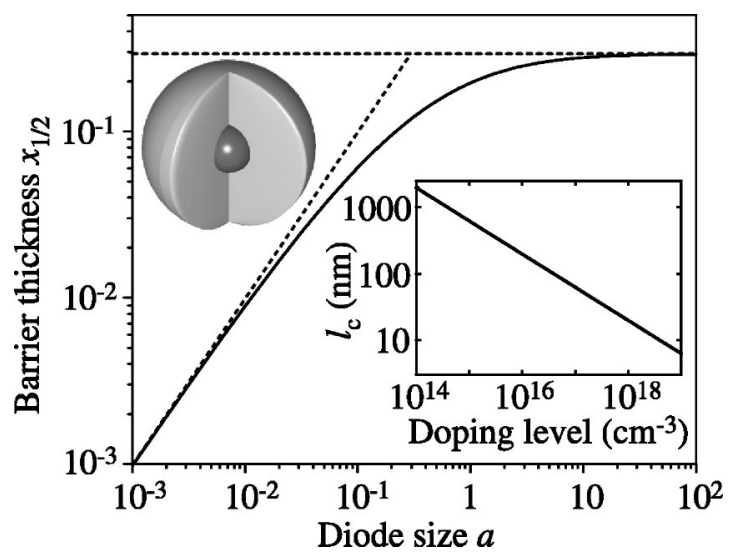

FIG. 1. Plot of the calculated barrier FWHM $x_{1 / 2}$ as a function of diode size $a$ [based on Eq. (1)], both in units of $l_{\mathrm{c}}$. The dashed lines represent the asymptotic values for $a \gg l_{\mathrm{c}}$ (conventional diode) and $a \ll l_{\mathrm{c}}$ (new regime), respectively. The lower right inset is a plot of $l_{c}$ as a function of doping level $N_{\mathrm{d}}$ in silicon $\left(\varepsilon_{\mathrm{s}}=11.7\right)$ for $\varphi_{\mathrm{B}}=0.67 \mathrm{eV}$ and $T=300 \mathrm{~K}$. The upper left inset schematically shows the model system, a metallic sphere embedded in semiconductor. 
$-V$ (with $\varphi_{\mathrm{s}}=E_{\mathrm{c}}-E_{\mathrm{f}}$ ). Equation (1) is valid for small bias voltage $V$. The limited validity of the depletion approximation at finite temperatures only affects the tail of the barrier (where $|V(x)| \leq k T$ ), which is unimportant for the transport properties. From the equation, it can be seen that the characteristic length scale of this system is

$$
l_{\mathrm{c}}=L_{\mathrm{D}} \sqrt{2 e V_{\mathrm{s}} / k T}=\sqrt{\frac{2 \varepsilon_{\mathrm{s}} V_{\mathrm{s}}}{e N_{\mathrm{d}}}} .
$$

By comparing the diode size $a$ to $l_{\mathrm{c}}$ we can decide whether the diode is "small" or "large." In the lower right inset of Fig. 1 the value of $l_{\mathrm{c}}$ is plotted versus doping concentration $N_{\mathrm{d}}$.

An important quantity for electrical transport is the Schottky barrier thickness. In Fig. 1, the barrier full width at half maximum (FWHM, $x_{1 / 2}$ ) calculated from Eq. (1), is plotted as a function of diode size $a$. From the figure it is clear that for $a \gg l_{\mathrm{c}}$ the value of $x_{1 / 2}$ approaches a constant, which was expected for a large diode. Indeed, for $a \gg l_{\mathrm{c}}$, Eq. (1) reduces to $V(x)=-\left(e N_{\mathrm{d}} / 2 \varepsilon_{\mathrm{s}}\right)(x-w)^{2}$, which is the well-known textbook ${ }^{11}$ result for band bending in the depletion approximation for an infinitely large diode. Both the depletion width $w=\sqrt{\left(2 \varepsilon_{\mathrm{s}} / e N_{\mathrm{d}}\right) V_{\mathrm{s}}}$ and $x_{1 / 2}$ are in that regime independent of $a$.

Figure 1 shows that for $a \leq l_{\mathrm{c}}$ the value of $x_{1 / 2}$ is no longer constant, but decreases with decreasing $a$. For $a \ll l_{\mathrm{c}}$ it approaches $x_{1 / 2}=a$, that is, the barrier thickness equals the diode size. This also follows from Eq. (1), which reduces to $V(x)=V_{\mathrm{s}} \cdot a /(a+x)$ for $a \ll l_{\mathrm{c}}$ and $x \ll w$ (that is, close to the interface). Note that this is exactly the potential due to the charged sphere only. In this regime, the effect of the semiconductor space charge on the barrier shape and thickness can be neglected. This can be understood from the fact that the screening due to the space charge region takes place on a length scale $l_{\mathrm{c}}$, as in conventional (large) diodes. However, from Gauss's law it follows that any charged object of typical size $d<\infty$ in a dielectric medium gives rise to a potential that behaves roughly as $V(r) \propto d / r$. This Coulomb potential can be further screened by the formation of a space charge layer of opposite sign, but that additional screening can be neglected if $d \ll l_{\mathrm{c}}$. This observation holds for any interface with typical dimensions much smaller than $l_{\mathrm{c}}$.

In a geometry that can actually be fabricated, the Poisson equation must be solved numerically. We have done this for $n$-doped silicon $\left(N_{\mathrm{d}}=10^{15} \mathrm{~cm}^{-3}\right)$ in contact with metallic circular disks of various radii. In all further calculations $\varphi_{\mathrm{B}}$ $=0.67 \mathrm{eV}$ was used, which is the barrier height of the $\mathrm{CoSi}_{2} / \mathrm{Si}(111)$-interface. ${ }^{12}$ Figure 2 shows the FWHM contours of the barriers as resulting from these calculations. Also shown are the FWHM contours of the barrier due to the metallic contacts only, illustrating the negligible effect of the space charge region on the barrier thickness in very small diodes. $^{13}$

To study the effect of the reduced barrier width on the transport properties of a small Schottky diode, a transmission coefficient $T(E, V)$ was obtained for the barrier shape from Eq. (1). This was done in a one-dimensional fully quantum mechanical calculation. ${ }^{14}$ Note that $T(E, V)$ is implicitly dependent on temperature and doping level, because these

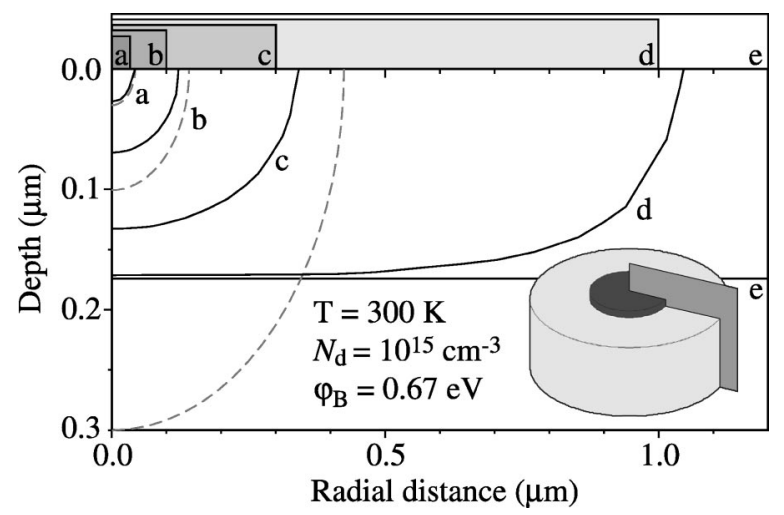

FIG. 2. The solid lines are contours of the barrier FWHM for various diskshaped contacts [see inset; radii ranging from $30 \mathrm{~nm} \mathrm{(a)} \mathrm{to} \mathrm{infinite} \mathrm{(e)],}$ taken from a numerical solution of the Poisson equation in silicon. It clearly shows the contact size dependence for contact radii smaller than $l_{\mathrm{c}}$ $\sim 750 \mathrm{~nm}$. The dashed lines are the FWHM contours of the barrier for the three smallest diodes, neglecting the screening effect of the semiconductor space charge region. The inset indicates the plane of cross section shown in the figure.

quantities influence the position of the Fermi level in the bulk semiconductor. The current density is then given by

$$
J(V) \propto \int_{0}^{\infty} T(E, V)\left[f\left(\varphi_{\mathrm{s}}+E\right)-f\left(\varphi_{\mathrm{s}}+E+V\right)\right] \mathrm{d} E,
$$

from which it follows that the zero bias differential conductance satisfies

$$
\left.\frac{\mathrm{d} J}{\mathrm{~d} V}\right|_{V=0} \propto-\int_{0}^{\infty} T(E, V) f^{\prime}\left(\varphi_{\mathrm{s}}+E\right) \mathrm{d} E .
$$

Here, $f$ is the Fermi-Dirac distribution function and $E$ the energy above the semiconductor conduction band edge. Transport due to electrons at energies below the barrier maximum $\left(E<V_{\mathrm{s}}\right)$ is regarded as tunneling, while for $E$ $>V_{\mathrm{s}}$ we speak of thermionic emission. Obviously, the contribution of thermionic emission is almost independent of the barrier thickness, while tunneling is strongly dependent on the barrier thickness.

In Fig. 3, the calculated zero bias differential conduction is plotted as a function of diode size $a$ for several values of $N_{\mathrm{d}}$. For $a \gtrsim l_{\mathrm{c}}$ this quantity is independent of $a$. For smaller

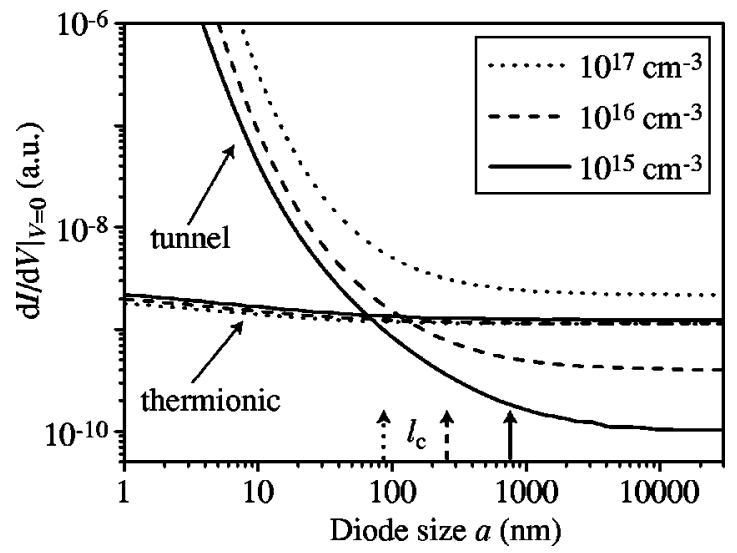

FIG. 3. The contributions of tunneling and thermionic emission to the zero bias differential conductance, plotted as a function of diode size $a$ for various doping concentrations. The vertical arrows indicate the values of $l_{\mathrm{c}}$. The parameters are the same as in Fig. 2. 


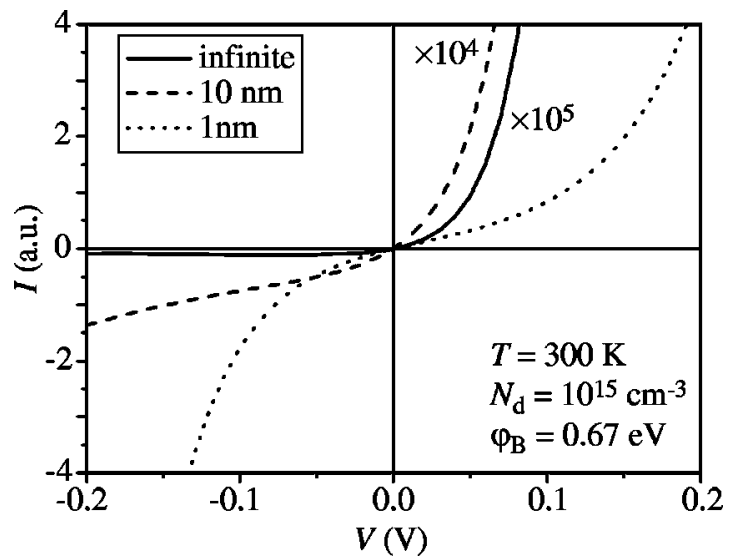

FIG. 4. Calculated $I V$ curves for various diode sizes. The large diode curve has the expected exponential shape. The qualitative appearance of the curves changes drastically with decreasing diode size. The curves of the larger diodes have been scaled vertically.

values, the tunnel current starts to increase rapidly, eventually leading to a strong increase of the total conduction.

Moreover, the shape of the $I V$ curves changes with decreasing diode size. Our calculations (Fig. 4) show that for large diodes the $I V$ curve has exactly its expected exponential shape $\{I \propto[\exp (e V / k T)-1]\}$. Apart from the total current increase, in small diodes the relative contribution of the reverse current starts to increase and eventually-in extremely small diodes- the reverse current exceeds the forward current, thus reversing the rectifying behavior of the diode.

Note that the image charge effect ${ }^{11}$ has been neglected so far. However, inclusion of this effect would only enhance the phenomenon mentioned previously as it reduces the effective barrier height and width even further, especially in barriers which are narrow already.

One more issue that needs to be discussed is that of discrete random dopants. In our analysis, the dopants played a role in determining the Fermi-level position in the semiconductor bulk and were considered to provide a homogeneous space charge region. However, for the realistic parameters $N_{\mathrm{d}}=10^{15} \mathrm{~cm}^{-3}$ and $a=30 \mathrm{~nm}$ (so $a \ll l_{\mathrm{c}}$ ), the volume in which the potential drops to half its initial value contains approximately one doping atom. Discrete energy levels of such a doping atom cannot be resolved at room temperature.
More importantly, the potential well due to an ionized single dopant will locally distort the barrier shape. This effect complicates the potential landscape, but it can only significantly increase the conduction of the diode, when the dopant resides close to the interface. ${ }^{15}$

In conclusion, we have shown by means of a simple electrostatic argument that the Schottky barrier thickness becomes a function of the diode size for small diodes (e.g., smaller than $l_{\mathrm{c}} \approx 80 \mathrm{~nm}$ for $N_{\mathrm{d}}=10^{17} \mathrm{~cm}^{-3}$ ). Consequently, the contribution of tunneling to the total conductance is greatly enhanced in small diodes. This effect explains several experimental results, ${ }^{3,4}$ without the assumption of a reduced SBH. Moreover, small diodes show $I V$ curve shapes that qualitatively differ from those of conventional diodes.

We wish to thank J. Caro and H. W. M. Salemink for detailed discussions concerning this work. This work is part of the research program of the "Stichting voor Fundamenteel Onderzoek der Materie (FOM)," which is financially supported by the "Nederlandse Organisatie voor Wetenschappelijk Onderzoek (NWO)." One of us (S.R.), wishes to acknowledge fellowship support from the Royal Netherlands Academy of Arts and Sciences.

${ }^{1}$ Z. Yao, H. W. C. Postma, L. Balents, and C. Dekker, Nature (London) 402, 273 (1999).

${ }^{2}$ Y. Cui and C. M. Lieber, Science 291, 851 (2001).

${ }^{3}$ P. Avouris, I.-W. Lyo, and Y. Hasegawa, J. Vac. Sci. Technol. A 11, 1725 (1993).

${ }^{4}$ R. Hasunuma, T. Komeda, and H. Tokumoto, Appl. Surf. Sci. 130-132, 84 (1998).

${ }^{5}$ F. Léonard and J. Tersoff, Phys. Rev. Lett. 83, 5174 (1999).

${ }^{6}$ A. A. Odintsov, Phys. Rev. Lett. 85, 150 (2000).

${ }^{7}$ R. T. Tung, Phys. Rev. B 45, 13509 (1992).

${ }^{8}$ C. Malagu, V. Guidi, M. Stefancich, M. C. Carotta, and G. Martinelli, J. Appl. Phys. 91, 808 (2002)

${ }^{9}$ T. Ioannides and X. Verykios, J. Catal. 161, 560 (1996).

${ }^{10}$ G. D. J. Smit, S. Rogge, and T. M. Klapwijk, Appl. Phys. Lett. 80, 2568 (2002).

${ }^{11}$ S. M. Sze, Physics of Semiconductor Devices, 2nd ed. (Wiley, New York, 1981).

${ }^{12}$ R. T. Tung, Mater. Chem. Phys. 32, 107 (1992).

${ }^{13}$ Note that the barrier shape is still implicitly dependent on $N_{\mathrm{d}}$ via $V_{\mathrm{s}}$ as it influences the Fermi-level position in the semiconductor band gap.

${ }^{14}$ J. S. Walker and J. Gathright, Am. J. Phys. 62, 408 (1994).

${ }^{15}$ The region where the potential lowering due to the Coulomb potential of the dopant exceeds $0.1 \mathrm{eV}$ has a radius of about $1 \mathrm{~nm}$. 